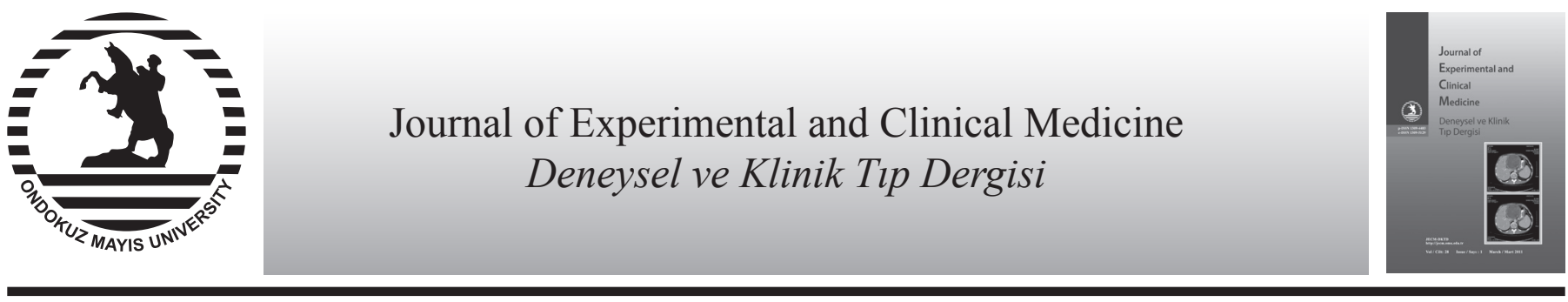

Case Report

\title{
Hypereosinophilic syndrome presenting as pruriginous erythroderma
}

\author{
Günseli Şefika Pancar*, Müge Güler Özden, Fatma Aydın, Nilgün Şentürk, M. Tayyar Cantürk, Ahmet Yaşar Turanlı \\ Department of Dermatology, Medical Faculty, Ondokuz Mayis University, Samsun, Turkey
}

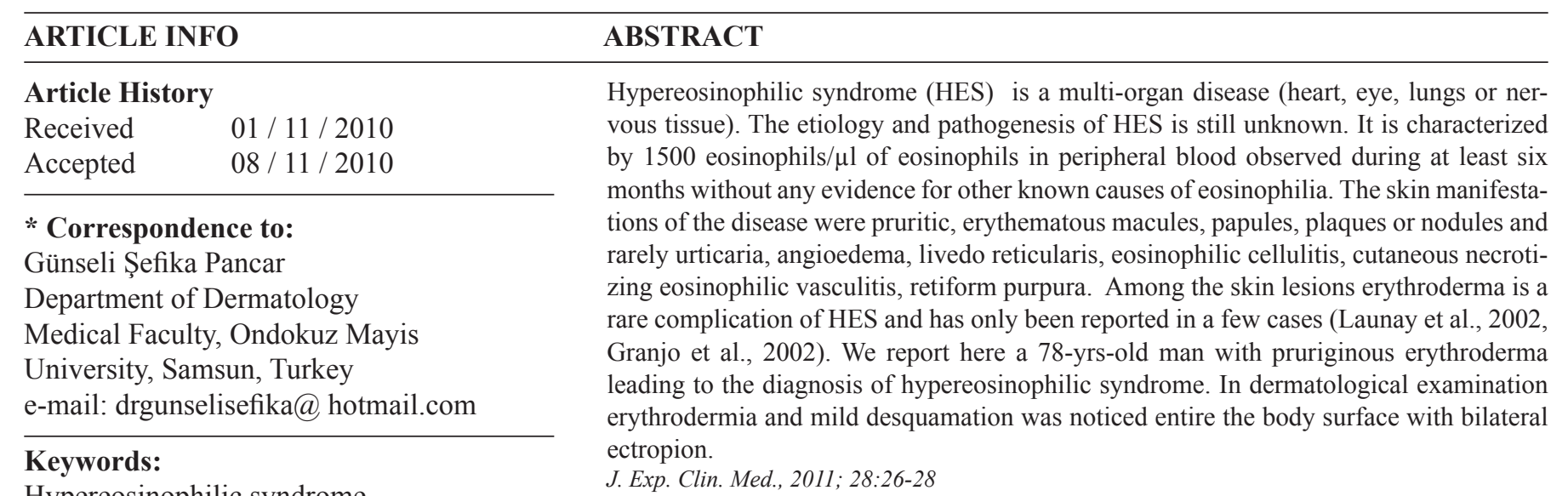

Hypereosinophilic syndrome

Erythroderma

Pruritus

Eosinophilia

Ectropion

Hydroxyurea

C 2011 OMU All rights reserved

\section{Introduction}

Hypereosinophilic syndrome (HES) is a multi-organ disease (heart, lungs or nervous tissue) characterized by 1500 eosinophils $/ \mu$ of eosinophils in peripheral blood observed during at least six months without any evidence for other known causes of eosinophilia. The skin findings include pruritic erythematous macules, papules and nodules, angioedema, urticarial plaques and erythroderma. Cutaneous lesions may be extremely debilitating and often reflect disease activity in HES (Leiferman et al., 2007). Erythroderma is one of the rare conditions of HES and there only a few cases in the literature. We report here a 78-yrs-old man with pruriginous erythroderma leading to the diagnosis of hypereosinophilic syndrome.

\section{Case Report}

A seventy eight-yrs-old male was admitted to our clinic with erythroderma in March, 2009. His previous history begun six years ago with an erythematous scaling pruritic plaque located on his left proximal extremity. The lesions spread to the whole body surface in this time interval. He had used topical and systemic steroids and acitretin because of the misdiagnosis as erythrodermic psoriasis in other clinics. In 2002 we first evaluated him with erythroderma, generalized desquamation and bilateral ectropion. White cell count was 13.3x109/1 and eosinophil level was 1900 eosinophils/ $\mu$ l. He was diagnosed as contact dermatitis and his lesions were partially healed after topical corticosteroid therapy. Two months later the patient referred with a relapsing condition and hospitalized for further investigation. We have recognized his weight loss and found that he has lost about $9 \mathrm{~kg}$ in the past 2 years. Besides he was using bronchodilator puffs for chronic obstructive pulmonary disease. He has no other medical history. Fever, pulse, blood pressure parameters were all in normal ranges. Physical examination is also normal except inguinal lympadenapathies $(1 \times 1 \mathrm{~cm})$. In dermatological examination erythrodermia and mild desquamation was noticed entire the body surface with bilateral ectropion (Fig. 1).

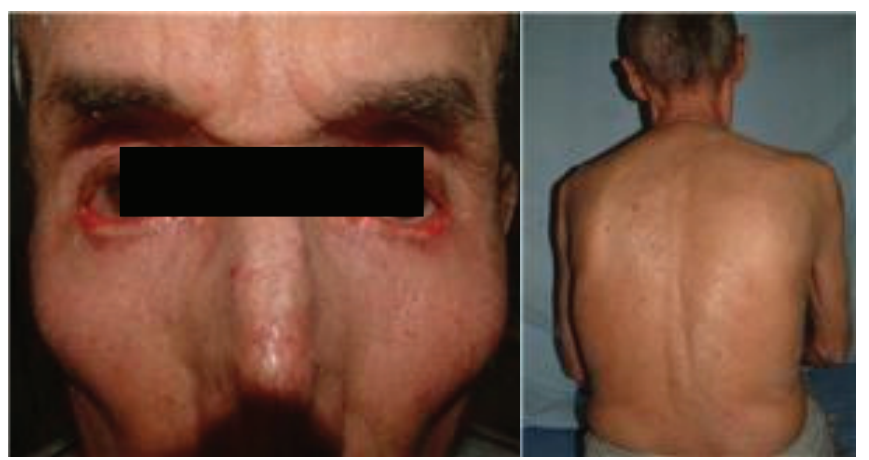

Fig. 1. Erythroderma with bilateral ectropion. 
Also dark hyperpigmented macular lesions with sharp margins were noticed on back and neck. Hyperkeratosis was prominent in palmar and plantar regions. Histopathologic examination of cutaneous lesions revealed dermal infiltrate of numerous eosinophils which forms microapses formation. Also eosinophils showed epidermotrophism and perivascular infiltration (Fig. 2).

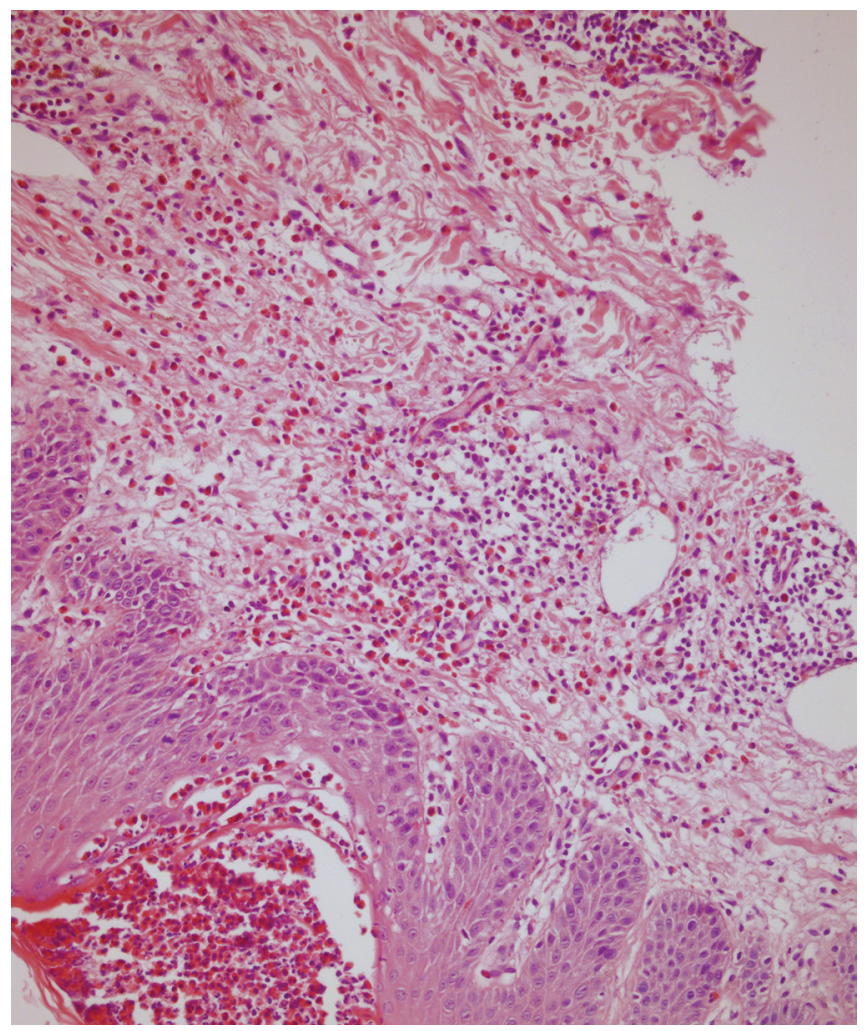

Fig. 2. Dermal infiltrate of numerous eosinophils which forms microapses formation (x100 H\&E).

Based on the sharp margined lesions that are located on his back and neck we peroformed microscopic examination of skin scrapings with $\mathrm{KOH}$ from whole body (trunk, back, extremities, neck, plantar and palmar region) and found very intensive fungal elements. Also trichophyton rubrum was determinated on culture but it could not be shown by histopathology with PAS stain.

Laboratory findings showed eosinophilic leukemia with 2900 eosinophils $/ \mu 1(22.3 \%$, normal range $0.9-6 \%)$. The examination of peripheral blood staining with Giemsa revealed significant eosinophilia $(>2 \%)$. Sezary cells in blood were negative. Ig E level was $1118 \mathrm{IU} / \mathrm{ml}$, ten times higher than the normal levels (5-120 IU/ml). Besides vitamin B 12 levels were higher than $1000 \mathrm{pg} / \mathrm{ml}$ (normal range 197-866 pg/ml). Hemoglobin and platelet levels were normal.

Analysis for parasitic infections was negative. Radiologic examination by computerized tomography revealed an infiltrative area in right sub pleural region of the lung. He had no fever or symptoms of pneumonia. We decided that eosinophils can be responsible with this infiltration. Cardiac involvement, thrombus formation and restrictive cardiomyopathy were not detected. Diastolic dysfunction was noticed by echocardiography.

The patient diagnosed as idiopathic hypereosinophilic syndrome. Because of the widespread dermatophytosis, hydroxyurea was choosed instead of systemic corticosteroid.
Terbinafine (topical and systemic forms) was started for dermatophyte treatment. Pruritus, erythroderma and desquamation were resolved, the sub pleural infiltrate was resolved and eosinophil values decreased to $1900 / \mu \mathrm{l}$ after eight weeks.

\section{Discussion}

In this article we report a patient with HES presenting as pruriginous erythroderma. Our patient has led to delay in diagnosis because of the misdiagnosis and patients's follow-up problems (psoriasis and contact dermatitis). Before the final diagnose we had decided that the reason for the pruriginous erythroderma was dermatophyte infection since we had found fungal elements at the body surface and trichophyton rubrum on culture. However this condition was not the reason but a complication. After we have treated this infection especially the sharp marginaed lesions healed but the pruritus, ectropion and eryhtoderma of the patient resisted. So the third reevaluation was made and resulted in the true diagnosis. The skin manifestations of the disease were pruritic, erythematous macules, papules, plaques or nodules and rarely urticaria, angioedema, livedo reticularis. However, some case reports have indicated that eosinophilic cellulitis, cutaneous necrotizing eosinophilic vasculitis, retiform purpura, livedo reticularis, superficial thrombophlebitis, Raynaud's phenomenon and digital gangrene may also occur as cutaneous features of HES (Tefferi et al., 2005).

Among the skin lesions erythroderma is a rare complication of HES and has only been reported in a few cases (Granjo et al., 2002; Launay et al., 2002). Eosinophilia greater than 1500/ul for more than 6 months, the lack of other known causes of eosinophilia, skin infiltration with eosinophils, hyper Ig E, Vitamin B12 grater than $1000 \mathrm{pg} / \mathrm{ml}$, skin lesions of plaques with erythrodermia and no vasculitis or granuloma formation on histopathology and regression with hydroxyurea treatment pointed to the diagnosis of HES. It was noted that eye involvement is a frequent and important feature of the hypereosinophilic syndrome

The principal cause of morbidity and mortality in HES is cardiac involvement. A restrictive type of cardiomyopathy such as endomyocardial fibrosis is characteristic. We did not determine cardiac involvement in our patient. The other dominant symptoms are hepatosplenomegaly, generalized lymphadenomegaly, myalgia, fever and retinal lesions (Granjo et al., 2002). Differential diagnoses are dermatophytosis with erythroderma, erythrodermic cutaneous T-cell lymphoma, atopic or contact erythroderma, churg strauss syndrome and drug eruption. Erythroderma and increased serum Ig E have been described in association with T-cell lymphomas however it was excluded by histopathology. He had no history of drug intake or contact sensitization. Although the microscopic findings pointed to the generalized erythrodermic tinea corporis infection, it was excluded by the histopathologic examination with eosinophils in microapses formation and negative PAS stain. The etiology and pathogenesis of HES is still unknown. It is speculative to think that this dermatophyte infection could play a role in pathogenesis since it seems a complication of topical and systemic corticosteroid treatments. Idiopathic hypereosinophilia is rare and is always diagnosed by exclusion of other diseases. The other differential diagnosis of HES includes Churg Strauss syndrome. Churg Strauss syndrome can resemble HES and also characterized 
with eosinophilia and hyper Ig E. Some authors considered to be an HES- associated disease (Tefferi et al., 2005; Leiferman et al., 2007). Characteristically patients with Churg strauss syndrome have asthma along with other organ involvement and exhibits vasculitis of the medium sized arteries and veins with extravascular granulomas in addition to the eosinophilic infiltrate. Also cutaneous lesions consist of hemorrhagic papules and nodules located on the scalp or extremities (Tefferi et al., 2005; Leiferman et al., 2007). All the symptoms of Churg strauss mentioned above were negative in our patient. So that findings pointed to the diagnosis of hypereosinophylic syndrome.

First line therapy for HES patients consists of prednisone at a dose of $1 \mathrm{mg} / \mathrm{kg} /$ day. Beside Prednisone also hydroxyurea and interferon alpha $2 b$ are the three major agents that allow control of eosinophilia. It has become evident that use of these agents in combination will control eosinophilia in most patients. Because of the widespread dermatophytosis, hydroxyurea which is an another excellent, cost-effective avenue was choosed instead of systemic steroids (Butterfield, 2007).

We report here a patient of HES presented as pruriginous erythroderma. This case is important since it emphasize a rare condition which causes eryhtroderma and the importance of reevaluation of such resistant patients.

\section{REFERENCES}

Butterfield, J.H. 2007. Treatment of hypereosinophilic syndromes with prednisone, hydroxyurea, and interferon. Immunol. Allergy Clin. North Am. 27, 493-518.

Granjo, E., Lima, M., Lopes, J.M. 2002. Chronic eosinophilic leukaemia presenting with erythroderma, mild eosinophilia and hyper-IgE: clinical, immunological and cytogenetic features and therapeutic approach. A case report. Acta. Haematol. 107, 108-112.

Launay D, Catteau B, Dubost-.Brama, A. 2002. A four-year history of pruriginous erythroderma leading to the diagnosis of idiopathic hypereosinophilic syndrome. Acta. Derm. Venereol. 82, 376-378.

Leiferman, K.M., Gleich, G.J., Peters, M.S. 2007.Dermatologic manifestations of the hypereosinophilic syndromes. Immunol. Allergy. Clin. North Am. 27, 415-441.

Tefferi, A. 2005. Modern diagnosis and treatment of primary eosinophilia. Acta. Haematol. 114, 52-56. 